IPMU16-0037

TUM-HEP-1039-16

\title{
LikeDM: likelihood calculator of dark matter detection
}

\author{
Xiaoyuan Huang ${ }^{1 *}$, Yue-Lin Sming Tsai ${ }^{2 \dagger}$, and Qiang Yuan ${ }^{3,4 \ddagger}$ \\ ${ }^{1}$ Physik-Department T30d, Technische Universität München, \\ James-Franck-Straße, D-85748 Garching, Germany \\ ${ }^{2}$ Kavli IPMU (WPI), University of Tokyo, Kashiwa, Chiba 277-8583, Japan \\ ${ }^{3}$ Department of Astronomy, University of Massachusetts, \\ 710 North Pleasant St., Amherst, MA, 01003, USA \\ ${ }^{4}$ Key Laboratory of Dark Matter and Space Astronomy, \\ Purple Mountain Observatory, Chinese Academy of Sciences, Nanjing 210008, China
}

(Dated: January 6, 2017)

With the large progress in searches for dark matter (DM) particles with indirect and direct methods, we develop a numerical tool that enables fast calculations of the likelihoods of specified DM particle models given a number of observational data, such as charged cosmic rays from space-borne experiments (e.g., PAMELA, AMS-02), $\gamma$-rays from the Fermi space telescope, and underground direct detection experiments. The purpose of this tool — LIKEDM, likelihood calculator for dark matter detection - is to bridge the gap between a particle model of DM and the observational data. The intermediate steps between these two, including the astrophysical backgrounds, the propagation of charged particles, the analysis of Fermi $\gamma$-ray data, as well as the DM velocity distribution and the nuclear form factor, have been dealt with in the code. We release the first version (v1.0) focusing on the constraints from indirect detection of DM with charged cosmic and gamma rays. Direct detection will be implemented in the next version. This manual describes the framework, usage, and related physics of the code.

\footnotetext{
* huangxiaoyuan@gmail.com

$\dagger$ smingtsai@gmail.com

$\ddagger$ yuanq@pmo.ac.cn
} 
PACS numbers: $95.35 .+\mathrm{d}, 96.50 . \mathrm{S}-$

\section{PROGRAM SUMMARY}

Program Title: LIKEDM

Licensing provisions: GPLv3

Programming language: FORTRAN 90 and Python

Operating system: Linux.

Nature of problem: Dealing with the intermediate steps between a dark matter model and data.

Solution method: Fast computation of the likelihood of a given dark matter model (defined by a mass, cross section or decay rate, and annihilation or decay yield spectrum), without digging into the details of cosmic-ray propagation, Fermi-LAT data analysis, or related astrophysical backgrounds.

\section{INTRODUCTION}

After the discovery of the $125 \mathrm{GeV}$ Higgs boson at the Large Hadron Collider [1, 2], we have a complete picture of the standard model (SM) of particle physics. The next step beyond the SM could be the identification of the dark matter (DM) particles that were suggested to be widely present in the Universe by a series of astronomical observations. Although the astronomical evidence could be attributed to gravitational interactions between DM and SM particles, we are yet to exclude the possibility of weakly interacting massive particles (WIMPs). The potential weak interactions between DM and SM particles provide us with the opportunity to identify DM, directly via collisions between DM particles and underground targets or indirectly via the products of DM annihilation or decay in the Universe. Many efforts have been made to find a direct signal of DM in an underground detector; however, no convincing evidence has been found till date [3-6]. On the other hand, with the operation of several new-generation space telescopes and detectors, such as PAMELA, AMS-02, and Fermi, many anomalies have been found in the high-energy sky [7-9]. The uncertainties from astrophysical backgrounds and/or astrophysical sources, however, make the identification of possible DM signals more challenging. Nevertheless, the constraints on 
DM models have become more and more stringent with the new direct and indirect data. Some of these constraints depend on certain assumptions about the backgrounds (e.g., the positron anomaly $[10,11])$. Since there is no consistent signal of DM present in all observations, we may expect that the assumptions of astrophysical contributions to those anomalies are reasonable. The combination of various kinds of observations is expected to give much improved constraints on DM models, which is one of the motivations for developing this tool for calculating the DM likelihood.

Another motivation is that it is non-trivial to confront DM models with observational data due to the complicated astrophysical backgrounds. First, a proper modeling of the backgrounds, with possible systematic uncertainties (e.g., the cosmic ray (CR) propagation parameters), is necessary when calculating the likelihood of a DM signal. Second, it is better to decouple the DM model inputs from the following astrophysical processes, as it enables our tool to be applied to any DM particle model. Third, we intend to have an efficient computation of the DM signal as well as the backgrounds. With these goals, we develop this likelihood calculator of DM detection, LIKEDM. The basic function of LIKEDM is to deal with the intermediate steps between a DM model and data. To achieve this goal, we 1) calculate the propagation of CR electrons/positrons and antiprotons with Green's functions with respect to energy (e.g., integrated with space and time), 2) model the CR backgrounds with phenomenological forms, 3) model the $\gamma$-ray emission with standard Fermi-LAT diffuse emission templates and point sources, and 4) calculate the likelihood map of $\gamma$-rays on the "energy-flux" plane for given regions of interest (ROIs). Some works have been published based on parts of these methods [12, 13]. Here we present the first version of this tool and make the code publicly available in the community and summarize the details in this manual. Constraints from direct detection have not been included in this release, and will be added in the subsequent version.

This manual is structured as follows. In Sec.II, we describe the calculation of charged CRs from both the DM signal and the background. The Green's function for fast computation of the propagation of charged CRs is presented. In Sec. III, we describe the likelihood calculation from Fermi-LAT observations of dwarf spheroids (dSphs). We give the energyflux likelihood map with updated Fermi-LAT data. We introduce the code, installation procedure, and explain the usage of LIKEDM in Sec. IV] Finally, we summarize in Sec. V] 


\section{CHARGED COSMIC RAYS}

\section{A. Propagation of charged cosmic ray particles}

The charged cosmic rays (CRs) propagate diffusively in the random magnetic field of the Milky Way. The interaction with the interstellar medium (ISM) will result in energy losses and/or fragmentation of the primary CRs, as well as the production of secondary CRs. For electrons/positrons, there will be additional energy losses due to radiation in the interstellar radiation field (ISRF) and the magnetic field. The random shocks in the interstellar space

may reaccelerate the low-energy CR particles. There may also be convective transport of CRs as evidenced by the wide existence of galactic winds. The general propagation equation of CRs in the Milky Way can be written as [14]

$$
\begin{aligned}
\frac{\partial \psi}{\partial t} & =Q(\mathbf{x}, p)+\nabla \cdot\left(D_{x x} \nabla \psi-\mathbf{V}_{\mathbf{c}} \psi\right)+\frac{\partial}{\partial p} p^{2} D_{p p} \frac{\partial}{\partial p} \frac{1}{p^{2}} \psi \\
& -\frac{\partial}{\partial p}\left[\dot{p} \psi-\frac{p}{3}\left(\nabla \cdot \mathbf{V}_{\mathbf{c}} \psi\right)\right]-\frac{\psi}{\tau_{f}}-\frac{\psi}{\tau_{r}},
\end{aligned}
$$

where $\psi$ is the CR differential density per unit momentum interval, $Q(\mathbf{x}, p)$ is the source function, $D_{x x}$ is the spatial diffusion coefficient, $\mathbf{V}_{\mathbf{c}}$ is the convection velocity, $D_{p p}$ is the diffusion coefficient in momentum space, $\dot{p} \equiv \mathrm{d} p / \mathrm{d} t$ is the momentum loss rate, and $\tau_{f}$ and $\tau_{r}$ are timescales for fragmentation and radioactive decay, respectively. A homogeneous spatial diffusion coefficient $D_{x x}$ is assumed, and the rigidity dependence is assumed to be of a power-law form $D_{x x}=D_{0} \beta\left(R / R_{0}\right)^{\delta}$, with $\beta$ being the velocity of the particle and $\delta$ reflecting the property of the ISM turbulence. For Kolmogrov turbulence, we have $\delta=1 / 3$. The reacceleration is described by diffusion in momentum space. The momentum diffusion coefficient $D_{p p}$ can be related to the spatial diffusion coefficient $D_{x x}$ by [15]

$$
D_{p p} D_{x x}=\frac{4 p^{2} v_{A}^{2}}{3 \delta\left(4-\delta^{2}\right)(4-\delta) w},
$$

where $v_{A}$ is the Alfven speed, and $w$ characterizes the level of turbulence which can be absorbed in $v_{A}$. The CRs are assumed to be confined in an extended halo with characteristic height $z_{h}$, beyond which free escape is assumed. Thus, the major propagation parameters are $D_{0}, \delta, v_{A}, V_{c}$ and $z_{h}$.

The secondary-to-primary ratios, such as $\mathrm{B} / \mathrm{C}$ and $(\mathrm{Sc}+\mathrm{Ti}+\mathrm{V}) / \mathrm{Fe}$, and the unstableto-stable ratios of secondary particles, such as ${ }^{10} \mathrm{Be} /{ }^{9} \mathrm{Be}$ and ${ }^{26} \mathrm{Al} /{ }^{27} \mathrm{Al}$ are often used to 
determine the propagation parameters 15 18]. There are numerical codes to compute CR propagation in the galaxy, such as GALPROP ${ }^{1}$ [16] and DRAGON ${ }^{2}$ [19].

In this tool, we adopt GALPROP version $50^{3}$ to calculate the propagation of charged particles. We adopt six sets of propagation parameters, with $z_{h}$ varying from $2 \mathrm{kpc}$ to 15 $\mathrm{kpc}$, which reflect the major uncertainties in the propagation parameters [20]. All groups are consistent with the $\mathrm{B} / \mathrm{C}$ data as well as the Fermi diffuse $\gamma$-ray emission data [21].

TABLE I: Propagation parameters.

\begin{tabular}{ccccc}
\hline \hline & $D_{0}^{a}$ & $z_{h}$ & $v_{A}$ & $\delta$ \\
& $\left(10^{28} \mathrm{~cm}^{2} \mathrm{~s}^{-1}\right)$ & $(\mathrm{kpc})$ & $\left(\mathrm{km} \mathrm{s}^{-1}\right)$ & \\
\hline 1 & 2.7 & 2 & 35.0 & 0.33 \\
2 & 5.3 & 4 & 33.5 & 0.33 \\
3 & 7.1 & 6 & 31.1 & 0.33 \\
4 & 8.3 & 8 & 29.5 & 0.33 \\
5 & 9.4 & 10 & 28.6 & 0.33 \\
6 & 10.0 & 15 & 26.3 & 0.33 \\
\hline \hline
\end{tabular}

${ }^{a}$ Diffusion coefficient at $R=4 \mathrm{GV}$.

\section{B. Green's function of charged particle fluxes from DM}

The annihilation or decay of DM particles in the Milky Way halo will produce charged CRs such as positrons and antiprotons, which will experience diffusive propagation before reaching the Earth. The fluxes of the charged CRs depend on both the density profile of DM and the propagation parameters (especially the height of the propagation halo $z_{h}$ ). We will consider several common forms of DM density profile, including the Navarro-Frenk-White (NFW) profile [22]

$$
\rho_{\mathrm{NFW}}(r)=\frac{\rho_{s}}{\left(r / r_{s}\right)\left(1+r / r_{s}\right)^{2}}
$$

\footnotetext{
1 http://galprop.stanford.edu/

2 http://www.dragonproject.org/Home.html

3 For the recent updated version 54 please refer to http://sourceforge.net/projects/galprop/.
} 
the Einasto (EIN) profile [23]

$$
\rho_{\mathrm{EIN}}(r)=\rho_{s} \cdot\left[-\frac{2}{\alpha}\left(\left(\frac{r}{r_{s}}\right)-1\right)\right],
$$

and the isothermal (ISO) profile [24]

$$
\rho_{\text {ISO }}(r)=\frac{\rho_{s}}{1+\left(r / r_{s}\right)^{2}} .
$$

The profile parameters are given in Table \125].

The source function of the charged CRs for DM annihilation or decay is

$$
q(E, r)= \begin{cases}\frac{\langle\sigma v\rangle}{2 m_{\chi}^{2}} \frac{d N}{d E} \times \rho^{2}(r) \text { for annihilation } \\ \frac{1}{m_{\chi} \tau} \frac{d N}{d E} \times \rho(r) \text { for decay }\end{cases}
$$

where $m_{\chi}$ is the mass of the DM particle, $\langle\sigma v\rangle$ is the annihilation cross section, $\tau$ is the decay lifetime, and $d N / d E$ is the particle yield spectrum per annihilation or decay.

TABLE II: DM density profile parameters.

\begin{tabular}{cccc}
\hline \hline & $r_{s}$ & $\rho_{s}$ & $\alpha$ \\
& $(\mathrm{kpc})$ & $\left(\mathrm{GeV} \mathrm{cm}^{-3}\right)$ & \\
\hline NFW & 20 & 0.26 & N/A \\
EIN & 20 & 0.06 & 0.17 \\
ISO & 5 & 1.16 & N/A \\
\hline \hline
\end{tabular}

The traditional way to solve the propagation of the DM-induced charged particles is to incorporate the source term for a given DM particle model in the propagation equation (Eq. (11)). In order to isolate the DM particle model from the propagation calculation, we approximate the function $d N / d E$ with a series of Gaussian kernel functions

$$
\frac{d N}{d E} \approx \sum_{i} C_{i} K_{i}\left(E, E_{i}\right)=\sum_{i} \frac{C_{i}}{\sqrt{2 \pi} \sigma_{i}} \exp \left[-\frac{\left(E-E_{i}\right)^{2}}{2 \sigma_{i}^{2}}\right],
$$

where $E_{i}$ and $\sigma_{i}$ are respectively the central value and width of the $i$ th Gaussian kernel. We find that generally $\sigma_{i}=15 \% E_{i}$ results in a good approximation to most of the energy spectrum $d N / d E$, except when it has very distinct (e.g., monochromatic) spectral structures. The left panel of Fig. 1 shows an illustration of the kernel functions weighted by the coefficients $C_{i}$ for a given spectrum. We can then calculate the propagated spectrum of each 
kernel function, $G_{i}(E)$, which is the approximate Green's function with respect to energy $E$ (dashed lines in the right panel of Fig. 1). The total propagated spectrum can be obtained as

$$
\Phi(E) \approx \sum_{i} C_{i} G_{i}(E)
$$

As shown in the right panel of Fig. 1, the result from this Green's function method is in good agreement with the direct calculation of the propagation (red dots).
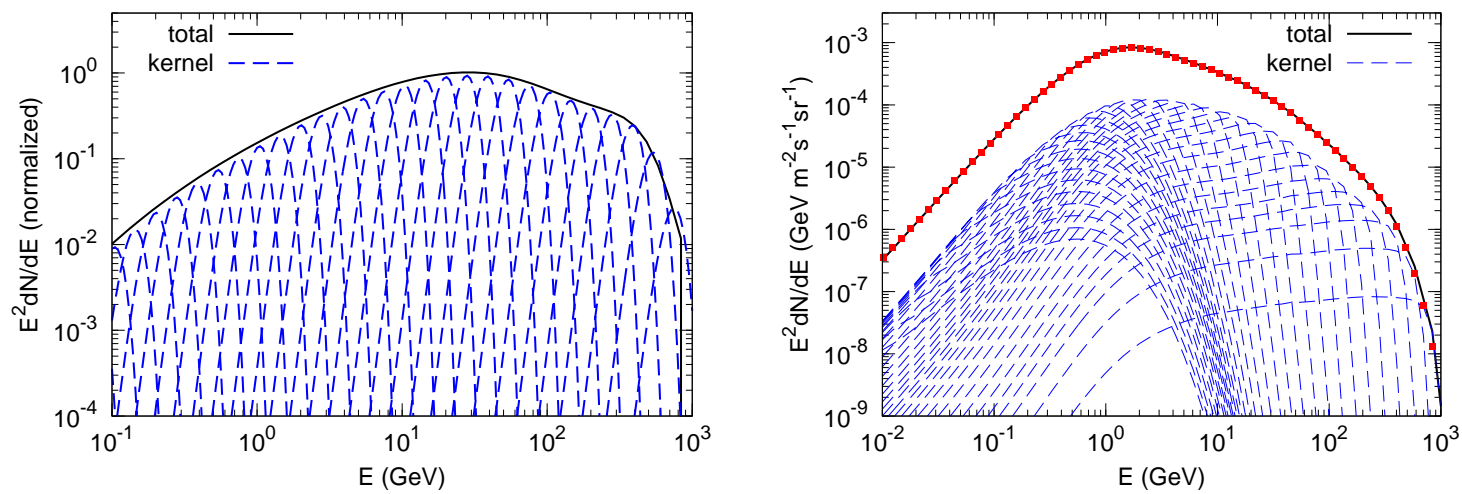

FIG. 1: Illustration of the kernel functions and the sum of the positron spectra, before (left) and after (right) the propagation. The points in the right panel show the direct calculation of the propagated spectrum of positrons with GALPROP. Here we adopt the second setting of the propagation parameters and the NFW profile of DM density. The mass of DM particle is $1 \mathrm{TeV}$, with an annihilation cross section of $\langle\sigma v\rangle=10^{-26} \mathrm{~cm}^{3} \mathrm{~s}^{-1}$ and $b \bar{b}$ as the annihilation final state.

Upon applying this method, any DM-induced CR $e^{ \pm}$and $\bar{p}$ spectra on Earth can easily be obtained by inserting its source shape. This helps us to significantly reduce the computation time ${ }^{4}$ if the predetermined Green's function tables are provided. Users are allowed to generate their own tables of Green's functions if necessary.

\section{Backgrounds}

The CR backgrounds relevant for the DM searches include the primary electrons from the CR sources, the secondary positrons and antiprotons from interactions between the primary

\footnotetext{
${ }^{4}$ As a rough example, the computation time of the propagation calculation reduces by a factor of $\gtrsim 10$ compared with the use of GALPROP.
} 
CR nuclei and the ISM, as well as the possible primary sources of positrons from e.g., pulsars [26]. Instead of using the more physical model which considers the injection/production and propagation of each type of particle [27, 28], we adopt an empirical model to fit the locally observed cosmic ray fluxes following Ref. [11]. This is equivalent to assuming that there is no DM "signal" in the current data and that all the measured events come from CR backgrounds (see also [29, 30]). In contrast to Ref. [11], we assume broken power-law forms to describe the fluxes of the primary electrons, secondary positrons/electrons and secondary antiprotons, with the purpose of reproducing the wide-band data:

$$
\begin{aligned}
\phi_{e^{-}} & =C_{e^{-}} E^{-\gamma_{1}^{-}}\left[1+\left(E / E_{\mathrm{br}}^{e^{-}}\right)^{\gamma_{2}^{e^{-}}}\right]^{-1}, \\
\phi_{e^{+}} & =C_{e^{+}} E^{-\gamma_{1}^{e^{+}}}\left[1+\left(E / E_{\mathrm{br}}^{e^{+}}\right)^{\gamma_{2}^{+}}\right]^{-1}, \\
\phi_{\bar{p}} & =C_{\bar{p}} E^{\gamma_{1}^{\bar{p}}}\left(1+E / E_{\mathrm{br}}^{\bar{p}}\right)^{-\gamma_{2}^{\bar{p}}} . .
\end{aligned}
$$

Note that the form of antiprotons is slightly different from that of electrons and positrons in order to improve the fit to the data. The secondary electron spectrum is assumed to be the same as the secondary positron spectrum, with a normalization factor of 0.6 as expected from the $p p$ collisions [31]. As for the extra source to reproduce the electron/positron excess, a power law with an exponential cut-off is assumed

$$
\phi_{s}=C_{s} E^{-\gamma_{s}} \exp \left(-E / E_{c}\right)
$$

Therefore the total fluxes of positrons, electrons, and positrons + electrons are

$$
\begin{aligned}
& \Phi_{e^{+}}=\phi_{e^{+}}+\phi_{s}, \\
& \Phi_{e^{-}}=\phi_{e^{-}}+0.6 \phi_{e^{+}}+\phi_{s}, \\
& \Phi_{e^{ \pm}}=\phi_{e^{-}}+1.6 \phi_{e^{+}}+2 \phi_{s},
\end{aligned}
$$

respectively, and the positron fraction is $\Phi_{e^{+}} / \Phi_{e^{ \pm}}$.

The data used to fit the backgrounds includes the updated AMS-02 positron fraction [32], the AMS-02 spectra of electrons and positrons [33], the AMS-02 total $e^{ \pm}$spectra [34], and the PAMELA antiproton spectrum [35]. The AMS-02 data below $1 \mathrm{GeV}$ are excluded from the fit [11]. The empirical background model gives a very good description of the data, as shown in Fig. 2. The best-fit $\chi^{2}$ value over the number of degrees of freedom (dof) is about $132.8 / 285$ for $e^{+} e^{-}$and about 11.1/19 for antiprotons. The best-fit parameters are listed in Table III. 
TABLE III: Best-fit parameters of the backgrounds.

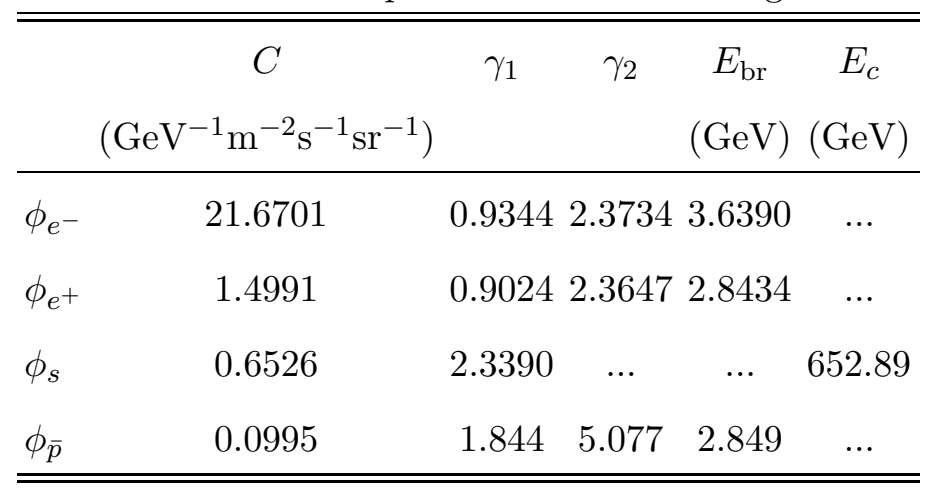

When a DM component is added to the model, we should allow for some freedom in the backgrounds to obtain a global best-fit to the data. Therefore, we multiply by factors of $\alpha_{i} E^{\beta_{i}}$, with $i=\left\{e^{-}, e^{+}, s, \bar{p}\right\}$, on the primary electrons, the secondary positrons/electrons, the extra positrons/electrons and the secondary antiprotons. We adopt the profile likelihood method to manage the nuisance parameters $\alpha_{i}$ and $\beta_{i}$, with the scan ranges $[0.1,10]$ and $[-0.5,0.5]$, respectively. The code Minuit [36] is used to find the maximum likelihood within the parameter space $\left[\alpha_{i}, \beta_{i}\right]$.

\section{Solar modulation}

The low-energy charged CRs will be modulated by solar activity. We adopt the simple force-field approximation with only one parameter, viz. the modulation potential, to calculate the effect of solar modulation [37]. Since our background model is an empirical one instead of a physical model, the solar modulation only applies to the CR fluxes from the DM annihilation or decay.

\section{E. The cosmic ray constraints on the DM annihilation parameters space}

In this subsection we present some results on the DM model parameter constraints from charged CRs derived with the above method. We adopt a DM annihilation scenario for illustration, and assume that the DM density profile is NFW. Given one set of the DM model parameters, such as the mass, the annihilation cross section, and the branching ratios to each annihilation channel, we calculate the production spectra of positrons and antiprotons 

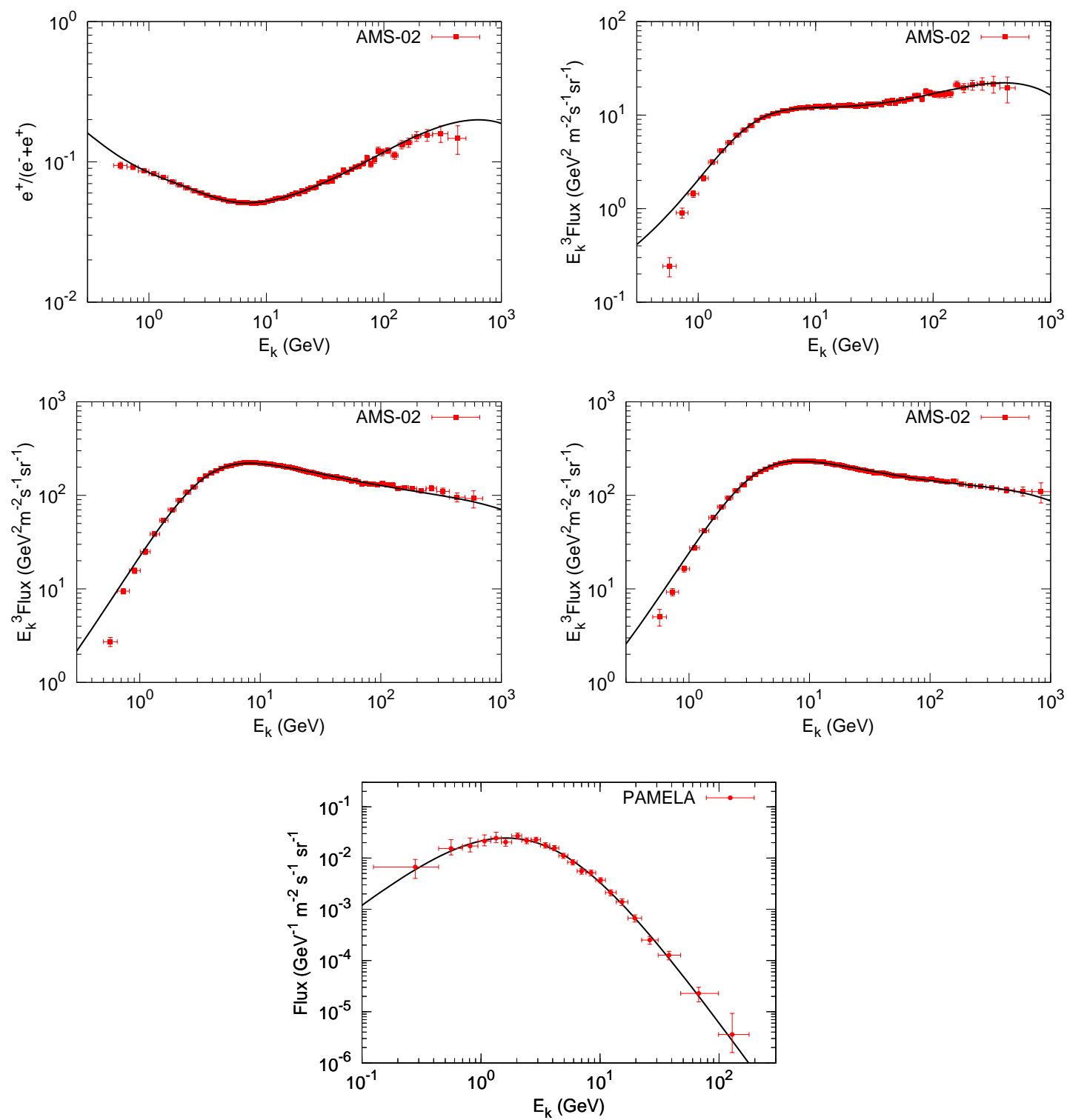

FIG. 2: Background fitting results of the positron fraction (top-left), positron (top-right), electron (middle-left), total $e^{ \pm}$(middle-right), and antiproton spectra (bottom).

using the tables ${ }^{5}$ of Ref. [38]. The propagated fluxes, calculated with the aforementioned Green's function method, together with the backgrounds, are then combined with the data to derive the likelihood, $\mathcal{L} \propto \exp \left(-\chi^{2} / 2\right)$, of this particular set of DM parameters.

The top-left panel of Fig. 3 shows a map of $-2 \Delta \ln (\mathcal{L}) \equiv-2 \ln \left(\mathcal{L} / \mathcal{L}^{0}\right)$, where $\mathcal{L}$ is the likelihood of the model with different values of $\langle\sigma v\rangle$ and $m_{\chi}$, and $\mathcal{L}^{0}$ is the likelihood for the

5 Only those tables of fluxes at production including EW corrections are used and incorporated in LIKEDM. 

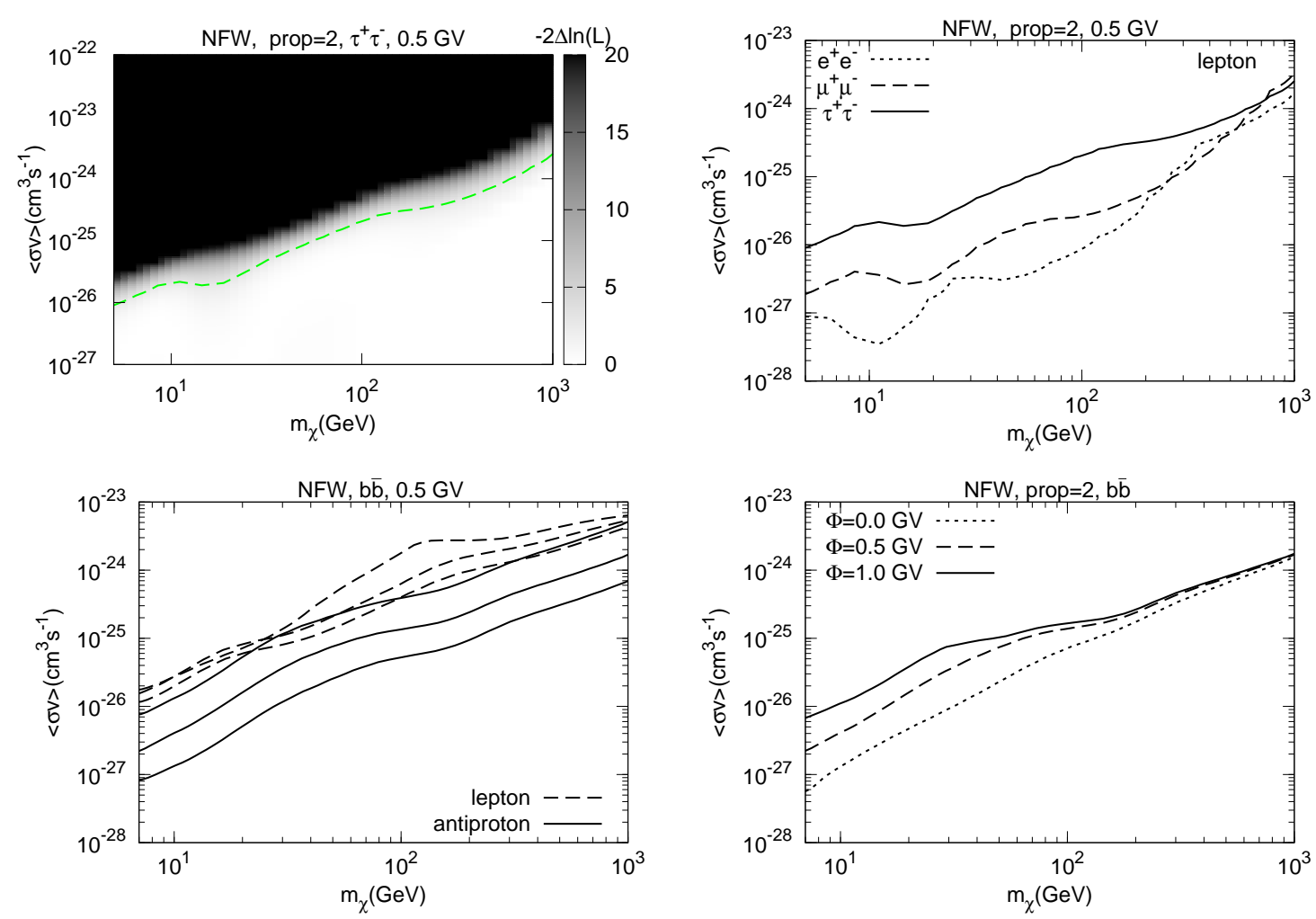

FIG. 3: Figure shows the constraints on the DM annihilation parameters from the charged CR data. The DM density profile is assumed to be NFW, and the solar modulation potential is adopted to be $0.5 \mathrm{GV}$ unless stated elsewhere. Top-left: the map of $-2 \Delta \ln (\mathcal{L})$ on the $\left(m_{\chi},\langle\sigma v\rangle\right)$ plane from the AMS-02 lepton data, for DM annihilation into $\tau^{+} \tau^{-}$. The dashed line shows the $95 \%$ CL limit. Top-right: $95 \%$ upper limits of $\langle\sigma v\rangle$ from the AMS-02 lepton data, for different DM masses and the annihilation channels of $e^{+} e^{-}, \mu^{+} \mu^{-}$, and $\tau^{+} \tau^{-}$, respectively. Bottom-left: $95 \%$ upper limits on $\langle\sigma v\rangle$ from the AMS-02 lepton data (dashed) and the PAMELA antiproton data (solid), for DM annihilation to $b \bar{b}$. The three lines of each group from top to bottom represent the propagation models \#1, \#2, and \#6, respectively. Bottom-right: $95 \%$ upper limits on $\langle\sigma v\rangle$ from the combination of the AMS-02 lepton data and the PAMELA antiproton data, for DM annihilation into $b \bar{b}$. The three lines from top to bottom represent the solar modulation potentials of $1.0,0.5$, and $0 \mathrm{GV}$, respectively.

null hypothesis (i.e., pure background). The likelihood is calculated using the AMS-02 $e^{+} e^{-}$ data. The propagation model is \#2, the solar modulation potential is $0.5 \mathrm{GV}$, and the DM annihilation channel is assumed to be $\tau^{+} \tau^{-}$. The dashed line shows the $95 \%$ confidence level 
(CL) upper limit, defined by $-2 \Delta \ln (\mathcal{L})=2.71$ for a single-sided probability distribution. Other panels of Fig. 3 illustrate the 95\% upper limits of the DM annihilation cross section for different channels (top-right), propagation models (bottom-left), and solar modulation potentials (bottom-right).

\section{GAMMA-RAYS FROM DSPHS}

Gamma-rays are another very important messenger for the indirect detection of DM. Gamma-rays travel through space without deflection, thus they can point back to the sources emitting them. It is advantageous to choose regions in the sky with high DM density and low astrophysical background to search for DM. The dSphs in the Milky Way are widely believed to be favorable targets with a high signal-to-noise ratio. Many works have been performed to search for DM-induced $\gamma$-rays from dSphs with Fermi-LAT data, yet none of them reported a significant detection [39-43]. Recently, the ongoing Dark Energy Survey (DES) reported some new candidates of dSphs in the southern hemisphere [44, 45]. Several groups had claimed possible weak $\gamma$-ray signals from Reticulum 2 [46] and Tucana III [47]. Since there are no reliable kinematic measurements available for these newly-discovered dSphs candidates (hence no reliable DM density profiles), the constraints and implications on DM from them are very uncertain. We adopt the dSphs sample of Ref. [42] in LIKEDM.

\section{A. Likelihood Map}

To ensure an easy computation of the total likelihood for any given shape of $\gamma$-ray spectrum, we take the likelihood map method first proposed in our earlier work [12] and further developed in Refs. [40, 42]. Briefly speaking, the likelihood $\mathcal{L}_{i j}$ of any flux $\phi_{j}$ in each energy bin $\left[E_{i-1 / 2}, E_{i+1 / 2}\right]$ is calculated to give a likelihood map on the $(E, \phi)$ plane. The total likelihood of a given spectrum can be simply obtained through a product of the likelihoods over all energy bins. This method is DM particle model independent, flexible and timesaving. Also, as shown in Ref. [48], it is simple to combine this method with data from other observations.

We describe the method in more detail. DM annihilation in dSphs is adopted for illustra- 
tion $^{6}$. The case of DM decay can be easily obtained via proper re-adjustment of the formula (see e.g., Eq. (6) ). The $\gamma$-ray flux from the annihilation of DM in a dSph is

$$
\phi(E)=\frac{\langle\sigma v\rangle}{8 \pi m_{\chi}^{2}} \times \frac{d N_{\gamma}}{d E_{\gamma}} \times J,
$$

where $J=\int d l d \Omega \rho(l)^{2}$ is the so-called $J$-factor which characterizes the amount of annihilation from a specified direction given the density distribution $\rho$ of DM. As the bin widths are small, for each energy bin $\left[E_{i-1 / 2}, E_{i+1 / 2}\right]$, we approximate $d N_{\gamma} / d E_{\gamma}$ with a constant, $C_{i}$. This approximation enables us to calculate the total $\log$-likelihood of the spectrum $\phi(E)$ from the logarithm of the likelihood map $\mathcal{L}_{i j}$ as

$$
\ln \mathcal{L}=\left.\sum_{i} \ln \mathcal{L}_{i j}\right|_{\phi_{j}=\frac{\langle\sigma v\rangle}{8 \pi m_{\chi}^{2}} \times J \times C_{i}} .
$$

We use the standard Fermi Science Tools package [52] version v10r0p5 to analyze the Fermi-LAT data. We use the newly released Fermi Pass 8 data, with four subsets of different point spread function (PSF) levels (i.e., PSF0, PSF1, PSF2 and PSF3), recorded from 4 August 2008 to 4 August 2015. These data are selected from $10^{\circ} \times 10^{\circ}$ box regions centered on each dSph, and $500 \mathrm{MeV}$ to $500 \mathrm{GeV}$ energies to reduce the impact from the bright Earth limb due to the large PSFs at low energies. The events with zenith angles greater than $100^{\circ}$ are also excluded. These selected data are divided into $100 \times 100$ spatial bins with $0.1^{\circ}$ bin size and 24 logarithmically spaced energy bins. Using the suggested diffuse background model ${ }^{7}$ including a structured Galactic component and an isotropic component, as well as point sources within $15^{\circ}$ of each dSph from the third Fermi catalog (3FGL; [49]) as astrophysical background, we first carry out a standard binned likelihood fitting over the entire energy range to get the best-fitting parameters for each point source and the diffuse components. Then we fix all the parameters of diffuse backgrounds and known point sources in the ROI, and add a point source at the position of the dSph. On varying the flux from the newly added point source, we calculate the $\ln \mathcal{L}_{i j}^{k l}$ for the $k$ th dSph and $l$ th subset of data in each energy bin and sum over $l$ to obtain the likelihood map $\mathcal{L}_{i j}^{k}$ for the $k$ th dSph.

\footnotetext{
${ }^{6}$ The decay case is also included in the code. Since the emission from DM decay is more extended, we use the profile parameters, which could get the median J-factor corresponding to $5^{\circ}$ radius integration, from the Markov Chain Monte Carlo (MCMC) fittings to the kinematic data of dSphs [50] to generate normalized two-dimensional SpatialMap. Then we calculate the likelihood map for these extended sources. For J-factors and corresponding errors please see Ref. [51].

7 http://fermi.gsfc.nasa.gov/ssc/data/access/lat/BackgroundModels.html
} 


\section{B. Combination of many dSphs}

If the $J$-factors of dSphs are known, then we can define a new variable, $\psi_{i}=\phi_{k}\left(E_{i}\right) / J_{k}=$ $\frac{\langle\sigma v\rangle}{8 \pi m_{\chi}^{2}} \times C_{i}$, and derive a combined log-likelihood map on the $(E, \psi)$ plane by adding the log-likelihoods of all dSphs together. Fig. 4 shows a combined log-likelihood map on the $\left(E, E^{2} \psi\right)$ plane, from the $15 \mathrm{dSphs}$ as listed in Ref. [42]. The $J$-factors of the dSphs are taken from Ref. [50]. The solid line shows the one-sided $95 \%$ confidence limit obtained from $-2 \Delta \ln \mathcal{L}_{i}=-2\left(\ln \mathcal{L}_{i}-\ln \mathcal{L}_{i}^{0}\right)=2.71$, where $\mathcal{L}_{i}^{0}$ is the likelihood for null-hypothesis (i.e., $\psi_{i}=0$ ) for the $i$ th energy bin. For any spectrum $\psi(E)$, the combined log-likelihood can be derived via a sum of log-likelihoods in all energy bins.

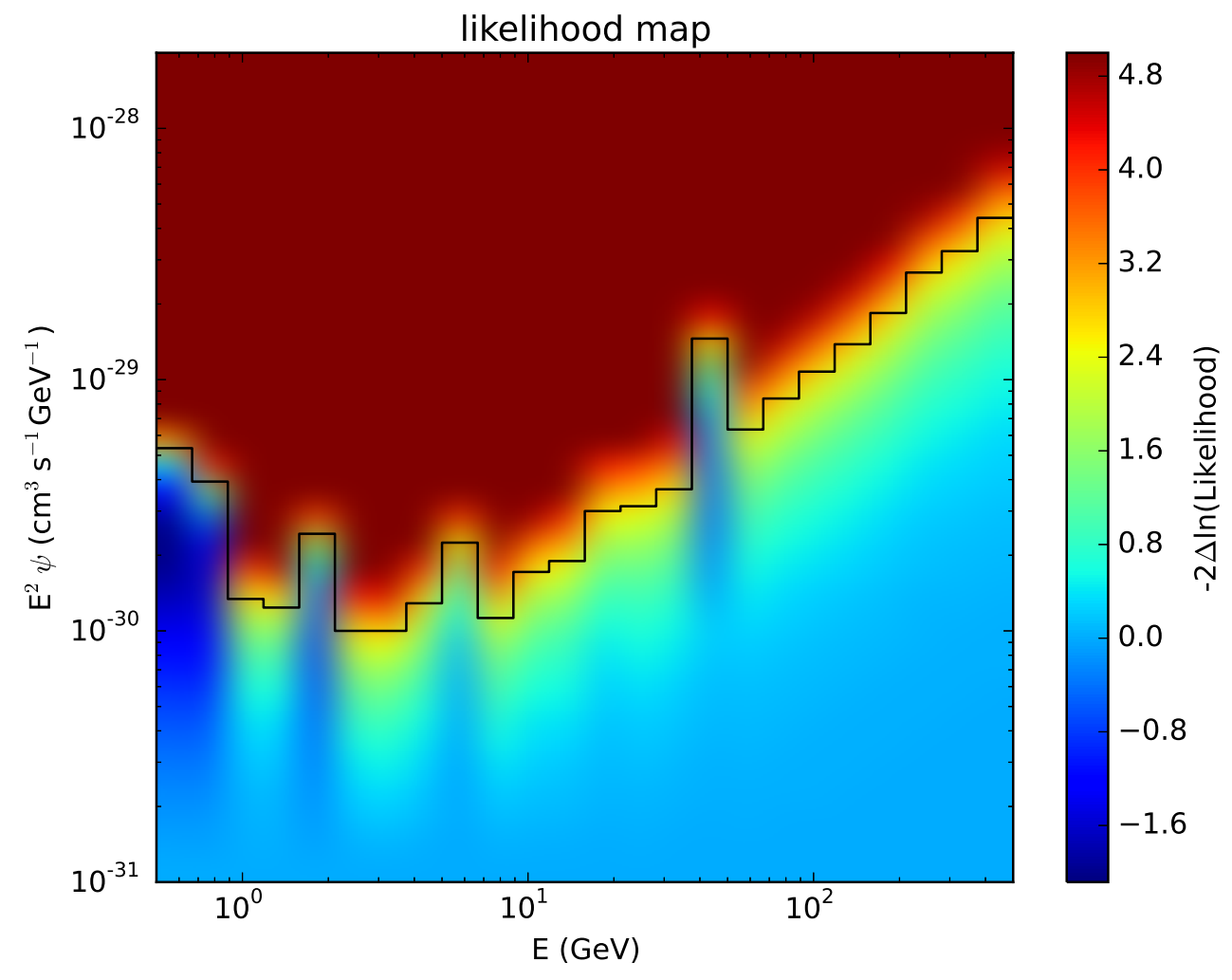

FIG. 4: The log-likelihood map on the $\left(E, E^{2} \psi\right)$ plane based on 7-year Fermi-LAT data of the 15 dSphs. The colors show the value of $-2 \Delta \ln \mathcal{L}$, normalized individually for each energy bin (see the text for details). The region above the solid line is excluded at the $95 \%$ confidence level.

However, in general the $J$-factors of dSphs cannot be well determined. If that is the case, we may not be able to have a combined likelihood map (such as that in Fig. 4) which 
is independent of $J$-factors ${ }^{8}$. We can define a likelihood term due to the uncertainties in $J$-factors as 42 ]

$$
\mathcal{L}_{J, k}\left(J_{\mathrm{obs}, k}, \sigma_{k}\right)=\frac{1}{\ln (10) J_{\mathrm{obs}, k} \sqrt{2 \pi} \sigma_{k}} e^{-\left[\log _{10}\left(J_{k}\right)-\log _{10}\left(J_{\mathrm{obs}, k}\right)\right]^{2} / 2 \sigma_{k}^{2}},
$$

where $k$ represents the $k$ th $\mathrm{dSph}, J_{k}$ is the "real" value of the $J$-factor and $J_{\mathrm{obs}, k}$ is the measured $J$-factor with error $\sigma_{k}$. The joint log-likelihood is then

$$
\ln \mathcal{L}(\text { Data } \mid \phi)=\sum_{k}\left(\left.\sum_{i} \ln \mathcal{L}_{i j}\right|_{\phi_{j}=\frac{\langle\sigma v\rangle}{8 \pi m \chi \chi} \times J \times C_{i}}+\ln \mathcal{L}_{J, k}\right) .
$$

Maximizing the above joint log-likelihood by varying $J_{k}$ for each $\mathrm{dSph}$, we can obtain the final log-likelihood of the spectrum $\phi(E)$.

In Fig. 5 we show the combined $95 \%$ upper limits for the $b \bar{b}$ annihilation channel. Here we adopt the $J$-factors given in Ref. [50]. The two solid lines show the differences between the cases with (green) and without (red) uncertainties in $J$-factor measurements. It shows the potential to improve the constraints with better determination of the $J$-factors.

\section{DESCRIPTION OF THE CODE}

In this section we describe the structure of the LIKEDM code. Users can download the source code from Ref. [53] or the batch file from the ancillary files to this paper on the arXiv website. LIKEDM is written in Fortran95, with a Python interface.

\section{A. Installation}

LikeDM uses the external package Minuit [36] to maximize the likelihoods, which needs to be installed first. To install pyLikeDM, the "f2py" package is required. We provide a BASH script (create_LikeDM.sh) for quick installation. After running create_LikeDM.sh, the user is prompted to enter a method of pyminuit installation:

./create_LikeDM.sh

${ }^{8}$ In Ref. [12] we profiled $J$-factors in the likelihood function for each energy bin, and obtained a combined likelihood map. However, this method multi-counts the $J$-factor uncertainties. A proper treatment should first combine likelihoods in different energy bins and then apply the $J$-factor likelihoods [40]. 


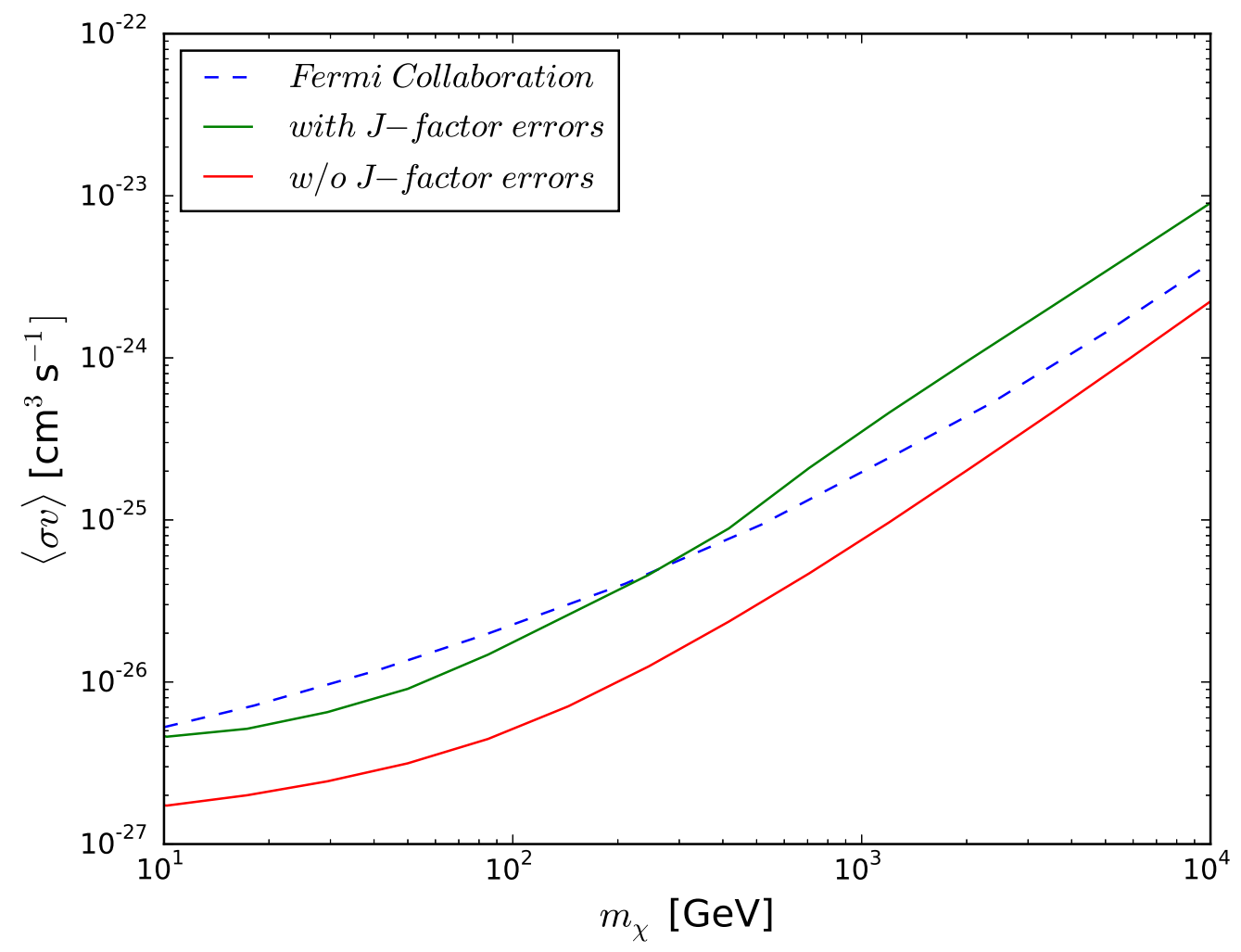

FIG. 5: $95 \%$ upper limits on the DM annihilation cross section for the $b \bar{b}$ annihilation channel, derived from a combined analysis of Fermi-LAT observations of 15 dSphs. The result obtained by Fermi-LAT collaboration with 6 year Pass 8 data is shown for comparison [42]. The two solid lines show the results with (green) and without (red) uncertainties of $J$-factor measurements.

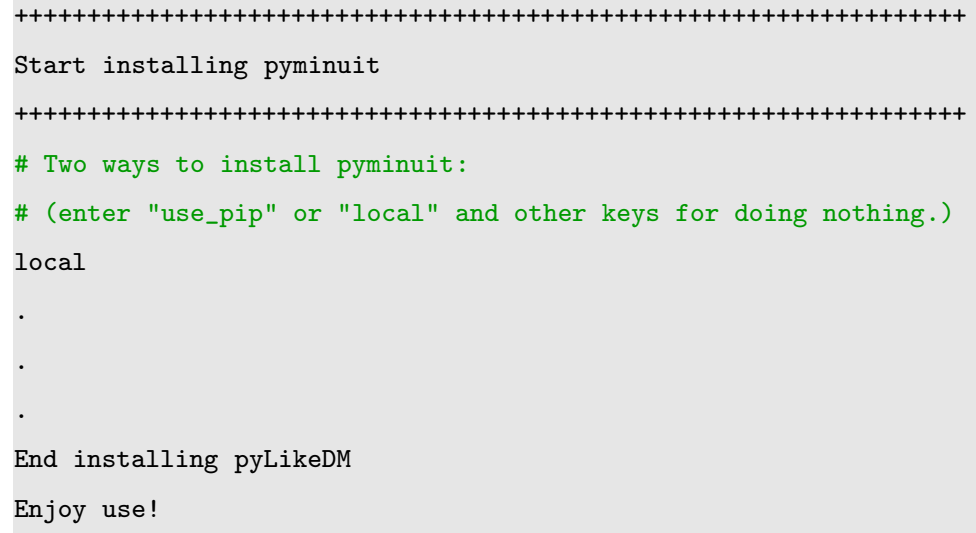

There are three options: use_pip, local, and any other key. If one chooses use_pip, sudo authorization is required to install iminuit via pip. If the user does not have pip 
installed, he/she can install pyminuit by using the local option. This step can be skipped if pyminuit has already been installed.

LIKEDM has been successfully installed and tested under Scientific Linux, Fedora, and Ubuntu operating systems.

\section{B. Running LikeDM}

The LIKEDM code can be called by

./pyLikeDM.py LikeDM_input_example.ini [dnde.spec]

where LikeDM_input_example.ini is an example file of the input parameters (see below part $\mathrm{C}$ for details), and the argument dnde.spec is optional, depending on the value of the logical parameter use_pppc4. If use_pppc4=T, then the DM annihilation or decay yield spectrum $d N / d E$ is computed using the PPPC4 tables [38]. Otherwise, the file dnde.spec with the spectrum generated by the user needs to be provided. The output looks like

LikeDM (version 1.0)

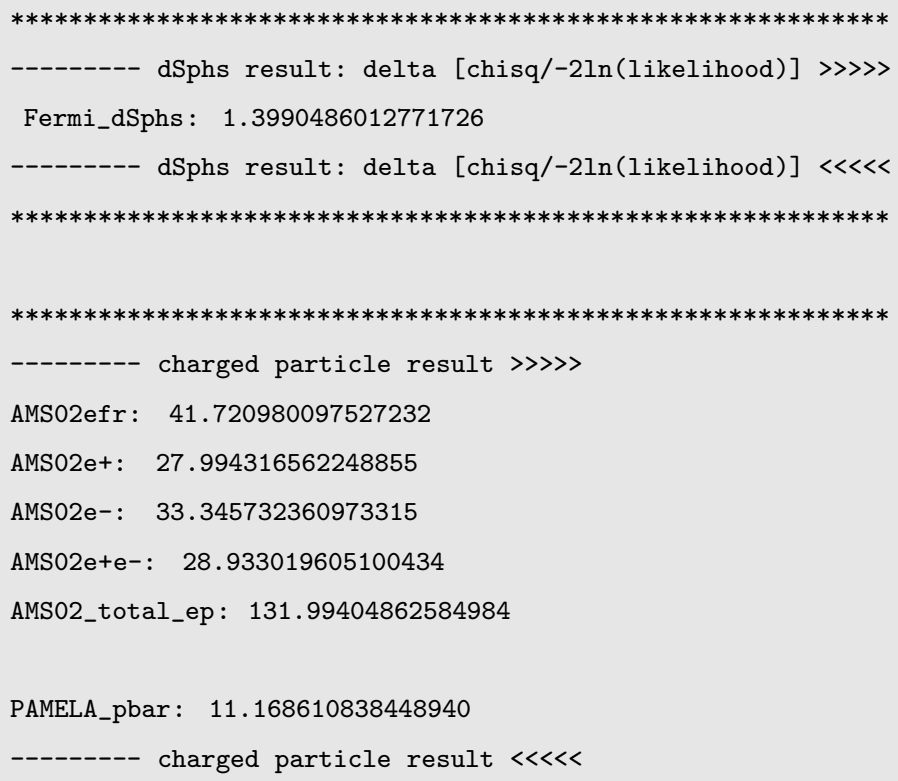




\section{Inputs and outputs}

We provide an example of the input file, LikeDM_input_example.ini, in the main folder of LIKEDM:

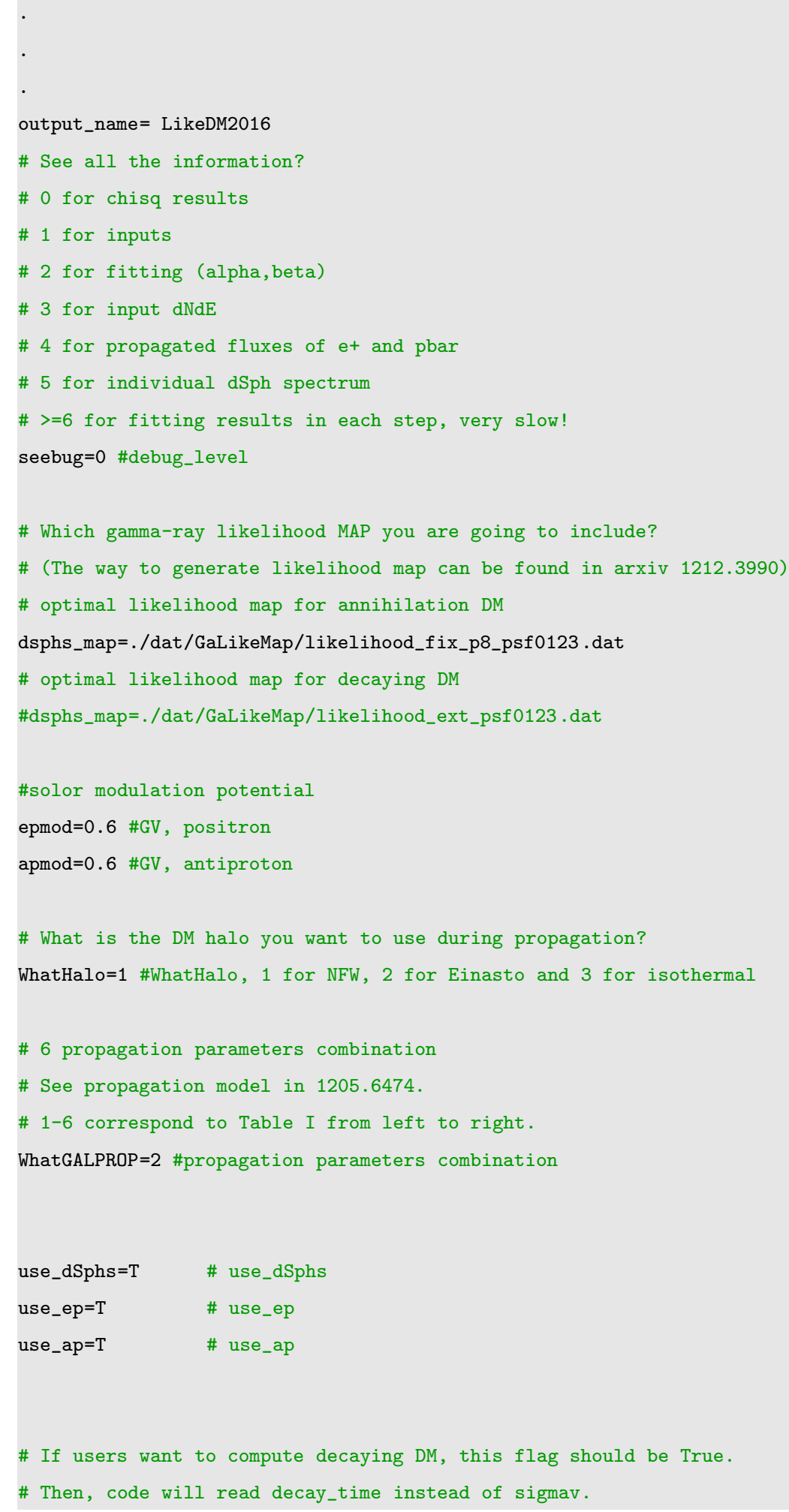




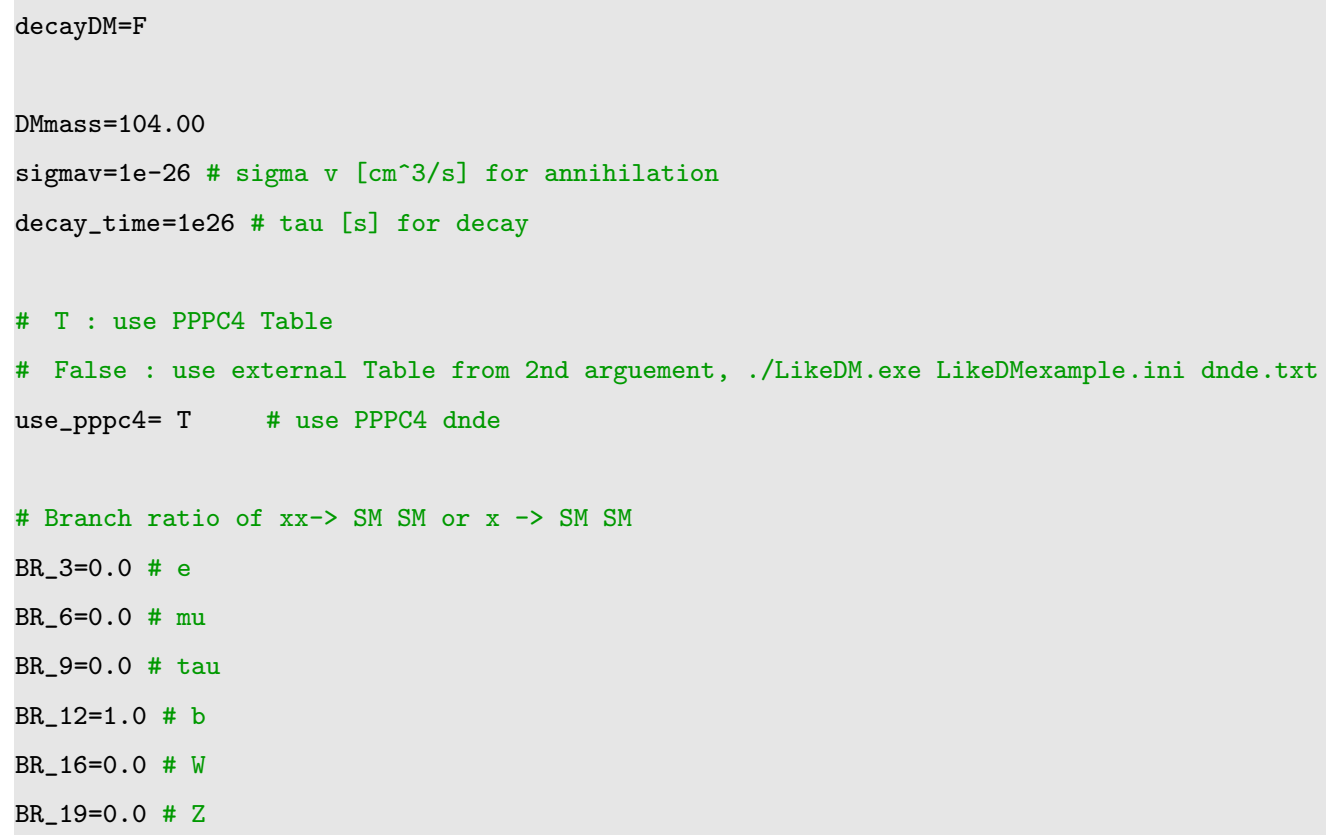

This input format is exactly the same as that in CosmoMC [54] and SuperBayes [55]. The modules to read the input file are src/Read_parameters.py and alternatively src/inifile.f90. The parameters are explained below:

\section{- output_name}

The name of the prefix of the output files. For the Python interface, this is not used because the output is shown on the screen. However, a user can always modify the subroutine print_debug_info to store the output with the name defined by this flag.

\section{- seebug}

An integer number to control the debug information shown on the screen. seebug=0 to 6 will print different kinds of results, for debugging or any interesting outputs such as the pre- or post-propagated particle spectra, and the fitting results of nuisance parameters.

- dsphs_map

Likelihood map of dSphs. The full path of the map is needed. For the case of decaying DM, the optimal likelihood map is recommended.

- epmod and apmod

Solar modulation potentials in units of GV, for electrons/positrons and protons/antiprotons, respectively. 
- WhatHalo

An integer number to specify the DM halo profile. 1 for NFW, 2 for Einasto and 3 for isothermal.

- WhatGALPROP

An integer number to determine the propagation parameters. 1 to 6 corresponds to the six sets of propagation parameters given in Ref. [20] (see also Table I of this manual).

- use_dSphs, use_ep, and use_ap

Logical flags to choose whether or not to use the corresponding data. The current version includes Fermi $\gamma$-ray data from dSphs, AMS-02 $e^{+} e^{-}$data, and PAMELA $\bar{p}$ data.

- decayDM

Logical flag to determine whether the DM annihilates or decays.

- DMmass, sigmav, decay_time

The DM mass in $\mathrm{GeV}$, annihilation cross section in $\mathrm{cm}^{3} \mathrm{~s}^{-1}$, and decaying lifetime in $\mathrm{s}$, respectively. sigmav takes effect when decayDM=F, and decay_time takes effects when decayDM=T.

- use_pppc4

Logical flag to specify whether to use the PPPC4 table to calculate $d N / d E$. If $\mathrm{F}$, an external file needs to be provided by the user. The file needs to be 4 columns, with E in $G e V,\left.\frac{d N}{d E}\right|_{\gamma}$ in $G e V^{-1},\left.\frac{d N}{d E}\right|_{e^{+}}$in $G e V^{-1}$, and $\left.\frac{d N}{d E}\right|_{\bar{p}}$ in $G e V^{-1}$, respectively.

- $B R \_x$

Branching ratios for different channels when using the PPPC4 table. The identification numbers can be found either at the PPPC4 website or in the beginning of the file src/PYTHIA_PPPC4.f90.

The outputs include the computed $\chi^{2}$ values on the screen. Users can easily modify the code src/monitorLikeDM.f90 to generate their own favored outputs or store the outputs to a file. 


\section{Package roadmap}

The source code of LIKEDM is located in the src/ folder. The main routine is pyLikeDM.py for the Python interface. We introduce the other routines grouped by their functionality:

- Initialization and Reading tables

src/ReadTable.f90

src/PYTHIA_PPPC 4 . f 90

src/inifile.f 90

The routine src/ReadTable.f90 reads the tables of the dSph likelihood map, the Green's functions for the propagation of positrons and antiprotons in the Galaxy, and the DM annihilation/decay spectra $d N / d E$ either from PPPC4 (connected with src/PYTHIA_PPPC4.f90) or the user supplied external file.

In addition to reading the tables, we also collect all the initialization subroutines in the src/ReadTable.f90 module and hence this module is the heart of LIKEDM.

The module src/inifile.f90 is taken from CosmoMC. It reads the parameter file and sets default values of the parameters. It is not used by default but a user can use this module if they wish to construct their own interface.

- Gamma-rays from dSphs

src/gamma_dSphs. $f 90$

This module provides the computation of DM annihilation/decay fluxes from a set of dSphs and their combined likelihood. The $J$-factors of these dSphs have been implemented in the likelihood calculation with a profile likelihood method. By default, a total of 15 dSphs, which are Bootes I, Canes Venatici II, Carina, Coma, Draco, Fornax, Hercules, Leo II, Leo IV, Sculptor, Segue I, Sextans, Ursa Major II, Ursa Minor, and Willman I, are included in the current version of LIKEDM. Users can enable or disable some dSphs likelihood by turning on/off the flags dsphs__use in 
the src/ReadTable.f90 module. The J-factors are taken from Ref. [50] for both annihilating and decaying DM.

- $\underline{\text { Charged cosmic rays: background }}$

src/charge_bkg.f 90

This routine calculates the background fluxes of $e^{+} e^{-}$and $\bar{p}$ using the empirical formulae described in Sec. II-C.

- $\underline{\text { Charged cosmic ray: DM } e^{+} \text {and } \bar{p}}$

src/charge_lepton. $f 90$

src/charge_antip. $f 90$

These two routines compute the propagated fluxes of positrons and antiprotons from DM annihilation or decay, using the Green's function method described in Sec. II-B.

- Charged cosmic rays: datasets

\section{src/charge_data. $f 90$}

This routine gives the cosmic ray data from AMS-02 [11, 32 34] and PAMELA [35], and returns the calculated $\chi^{2}$ values for given theoretical fluxes.

- Auxiliary module

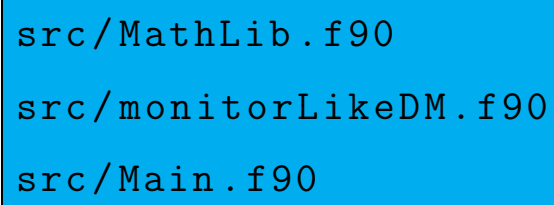

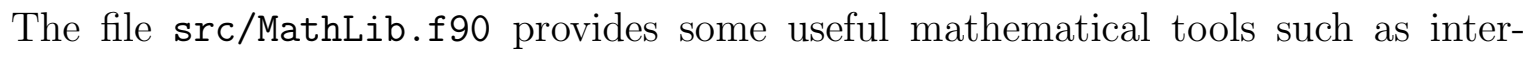
polation and integration. The routine src/monitorLikeDM.f90 gives the outputs controlled by the flag seebug. We also have a main routine, src/Main.f90, which is 
currently not used in the Python version but left as an alternative in the pure Fortran version.

\section{SUMMARY}

We present a publicly-available tool, LIKEDM, for likelihood calculations in DM models. It enables fast computation of the likelihood of a given DM model (defined by mass, cross section or decay rate, and annihilation or decay yield spectrum), without digging into the details of CR propagation, Fermi-LAT data analysis, or related astrophysical backgrounds. This code depends only on the Minuit minimization package, and is easy to install and run. The code LikEDM also provides an easy framework that can be linked to any particle model or Monte-Carlo code to perform a global study.

The currently released version (v1.0) contains only the indirect detection data, including the electron/positron measurements by AMS-02, the antiproton measurements by PAMELA, and the $\gamma$-ray observations from dSphs by Fermi-LAT. Further developments with more data, e.g., from the $\gamma$-ray observations of the Galactic center and isotropic background, as well as underground direct detection data, will be carried out soon.

\section{Acknowledgments}

We thank Vincent Bonnivard who kindly provides the MCMC results about the profile parameters of dSphs, and Andrew Fowlie and Shankha Banerjee who carefully read and improve the presentation of the manuscript. Y.S.T. was supported by World Premier International Research Center Initiative (WPI), MEXT, Japan. Q.Y. was supported by the National Key Program for Research and Development (No. 2016YFA0400200) and the 100 Talents program of Chinese Academy of Sciences.

[1] G. Aad et al. [ATLAS Collaboration], Phys. Lett. B 716, 1 (2012) [arXiv:1207.7214 [hep-ex]].

[2] S. Chatrchyan et al. [CMS Collaboration], Phys. Lett. B 716, 30 (2012) [arXiv:1207.7235 [hep-ex]]. 
[3] E. Aprile et al. [XENON100 Collaboration], Phys. Rev. Lett. 109, 181301 (2012) [arXiv:1207.5988 [astro-ph.CO]].

[4] D. S. Akerib et al. [LUX Collaboration], arXiv:1310.8214 [astro-ph.CO].

[5] Q. Yue et al. [CDEX Collaboration], Phys. Rev. D 90, 091701 (2014) [arXiv:1404.4946 [hep$\mathrm{ex}]]$.

[6] M. Xiao et al. [PandaX Collaboration], Sci. China Phys. Mech. Astron. 57, 2024 (2014) [arXiv:1408.5114 [hep-ex]].

[7] M. Cirelli, Pramana 79, 1021 (2012) [arXiv:1202.1454 [hep-ph]].

[8] L. Bergstrom, Annalen Phys. 524, 479 (2012) [arXiv:1205.4882 [astro-ph.HE]].

[9] X. J. Bi, P. F. Yin and Q. Yuan, Front. Phys. China 8, 794 (2013) [arXiv:1409.4590 [hep-ph]].

[10] O. Adriani et al. [PAMELA Collaboration], Nature 458, 607 (2009) [arXiv:0810.4995 [astro$\mathrm{ph}]$.

[11] M. Aguilar et al. [AMS Collaboration], Phys. Rev. Lett. 110, 141102 (2013).

[12] Y. -L. S. Tsai, Q. Yuan and X. Huang, JCAP 1303, 018 (2013) [arXiv:1212.3990 [astroph.HE]].

[13] A. Arhrib, Y. L. S. Tsai, Q. Yuan and T. C. Yuan, JCAP 1406, 030 (2014) [arXiv:1310.0358 [hep-ph]].

[14] A. W. Strong, I. V. Moskalenko and V. S. Ptuskin, Ann. Rev. Nucl. Part. Sci. 57, 285 (2007) [astro-ph/0701517].

[15] E. S. Seo and V. S. Ptuskin, Astrophys. J. 431, 705 (1994)

[16] A. W. Strong and I. V. Moskalenko, Astrophys. J. 509, 212 (1998) [astro-ph/9807150].

[17] D. Maurin, F. Donato, R. Taillet and P. Salati, Astrophys. J. 555, 585 (2001) [astro$\mathrm{ph} / 0101231]$.

[18] A. Putze, L. Derome and D. Maurin, Astron. Astrophys. 516, A66 (2010) [arXiv:1001.0551 [astro-ph.HE]].

[19] C. Evoli, D. Gaggero, D. Grasso and L. Maccione, JCAP 0810, 018 (2008) [arXiv:0807.4730 [astro-ph]].

[20] M. Ackermann et al. [LAT Collaboration], Astrophys. J. 761, 91 (2012) [arXiv:1205.6474 [astro-ph.CO]].

[21] M. Ackermann et al. [LAT Collaboration], Astrophys. J. 750, 3 (2012)

[22] J. F. Navarro, C. S. Frenk and S. D. M. White, Astrophys. J. 490, 493 (1997) [astro- 
$\mathrm{ph} / 9611107]$.

[23] J. Einasto, Trudy Astrofizicheskogo Instituta Alma-Ata 5, 87 (1965)

[24] J. N. Bahcall and R. M. Soneira, Astrophys. J. Suppl. 44, 73 (1980).

[25] G. Bertone, M. Cirelli, A. Strumia and M. Taoso, JCAP 0903, 009 (2009) [arXiv:0811.3744 [astro-ph]].

[26] C. S. Shen, Astrophys. J. 162, L181 (1970).

[27] Q. Yuan, X. J. Bi, G. M. Chen, Y. Q. Guo, S. J. Lin and X. Zhang, Astropart. Phys. 60, 1 (2015) [arXiv:1304.1482 [astro-ph.HE]].

[28] Q. Yuan and X. J. Bi, Phys. Lett. B 727, 1 (2013) [arXiv:1304.2687 [astro-ph.HE]].

[29] L. Feng, Q. Yuan, X. Li and Y. Z. Fan, Phys. Lett. B 720, 1 (2013) [arXiv:1206.4758 [astroph.HE]].

[30] L. Bergstrom, T. Bringmann, I. Cholis, D. Hooper and C. Weniger, Phys. Rev. Lett. 111, 171101 (2013) [arXiv:1306.3983 [astro-ph.HE]].

[31] T. Kamae, N. Karlsson, T. Mizuno, T. Abe and T. Koi, Astrophys. J. 647, 692 (2006) [Erratum-ibid. 662, 779 (2007)] [astro-ph/0605581].

[32] L. Accardo et al. [AMS Collaboration], Phys. Rev. Lett. 113, 121101 (2014).

[33] M. Aguilar et al. [AMS Collaboration], Phys. Rev. Lett. 113, 121102 (2014).

[34] M. Aguilar et al. [AMS Collaboration], Phys. Rev. Lett. 113, 221102 (2014).

[35] O. Adriani et al. [PAMELA Collaboration], Phys. Rev. Lett. 105, 121101 (2010) [arXiv:1007.0821 [astro-ph.HE]].

[36] F. James and M. Roos, Comput. Phys. Commun. 10, 343 (1975).

[37] L. J. Gleeson and W. I. Axford, Astrophys. J. 154, 1011 (1968).

[38] M. Cirelli, G. Corcella, A. Hektor, G. Hutsi, M. Kadastik, P. Panci, M. Raidal and F. Sala et al., JCAP 1103, 051 (2011) [JCAP 1210, E01 (2012)] [arXiv:1012.4515 [hep-ph]].

[39] M. Ackermann et al. [Fermi-LAT Collaboration], Phys. Rev. Lett. 107, 241302 (2011) [arXiv:1108.3546 [astro-ph.HE]].

[40] M. Ackermann et al. [Fermi-LAT Collaboration], Phys. Rev. D 89, 042001 (2014) [arXiv:1310.0828 [astro-ph.HE]].

[41] A. Geringer-Sameth, S. M. Koushiappas and M. G. Walker, Phys. Rev. D 91, no. 8, 083535 (2015) [arXiv:1410.2242 [astro-ph.CO]].

[42] M. Ackermann et al. [Fermi-LAT Collaboration], Phys. Rev. Lett. 115, no. 23, 231301 (2015) 
[arXiv:1503.02641 [astro-ph.HE]].

[43] Y. Zhao, X. J. Bi, H. Y. Jia, P. F. Yin and F. R. Zhu, arXiv:1601.02181 [astro-ph.HE].

[44] K. Bechtol et al. [DES Collaboration], Astrophys. J. 807, no. 1, 50 (2015) [arXiv:1503.02584 [astro-ph.GA]].

[45] A. Drlica-Wagner et al. [DES Collaboration], Astrophys. J. 813, no. 2, 109 (2015) [arXiv:1508.03622 [astro-ph.GA]].

[46] A. Geringer-Sameth, M. G. Walker, S. M. Koushiappas, S. E. Koposov, V. Belokurov, G. Torrealba and N. W. Evans, Phys. Rev. Lett. 115, no. 8, 081101 (2015) [arXiv:1503.02320 [astroph.HE]].

[47] S. Li et al., Phys. Rev. D 93, no. 4, 043518 (2016) [arXiv:1511.09252 [astro-ph.HE]].

[48] M. L. Ahnen et al. [MAGIC and Fermi-LAT Collaborations], JCAP 1602, no. 02, 039 (2016) [arXiv:1601.06590 [astro-ph.HE]].

[49] F. Acero, Astrophys. J. Suppl. 218, no. 2, 23 (2015).

[50] V. Bonnivard et al., Mon. Not. Roy. Astron. Soc. 453, no. 1, 849 (2015) [arXiv:1504.02048 [astro-ph.HE]].

[51] http://lpsc.in2p3.fr/clumpy/download/J_and_D_dsphs_CLs.tar.gz

[52] http://fermi.gsfc.nasa.gov/ssc/data/analysis/software/

[53] The code LikeDM can be downloaded from following websites.

https://likedm.hepforge.org

https://github.com/LikeDM/LikeDM_v1.0

http://www.space.pmo.cas.cn/aboutus/yuanqiang/likedm/201612/t20161226_358632.html

[54] A. Lewis and S. Bridle, Phys. Rev. D 66, 103511 (2002) [astro-ph/0205436].

[55] R. R. de Austri, R. Trotta and L. Roszkowski, JHEP 0605, 002 (2006) [hep-ph/0602028]. 


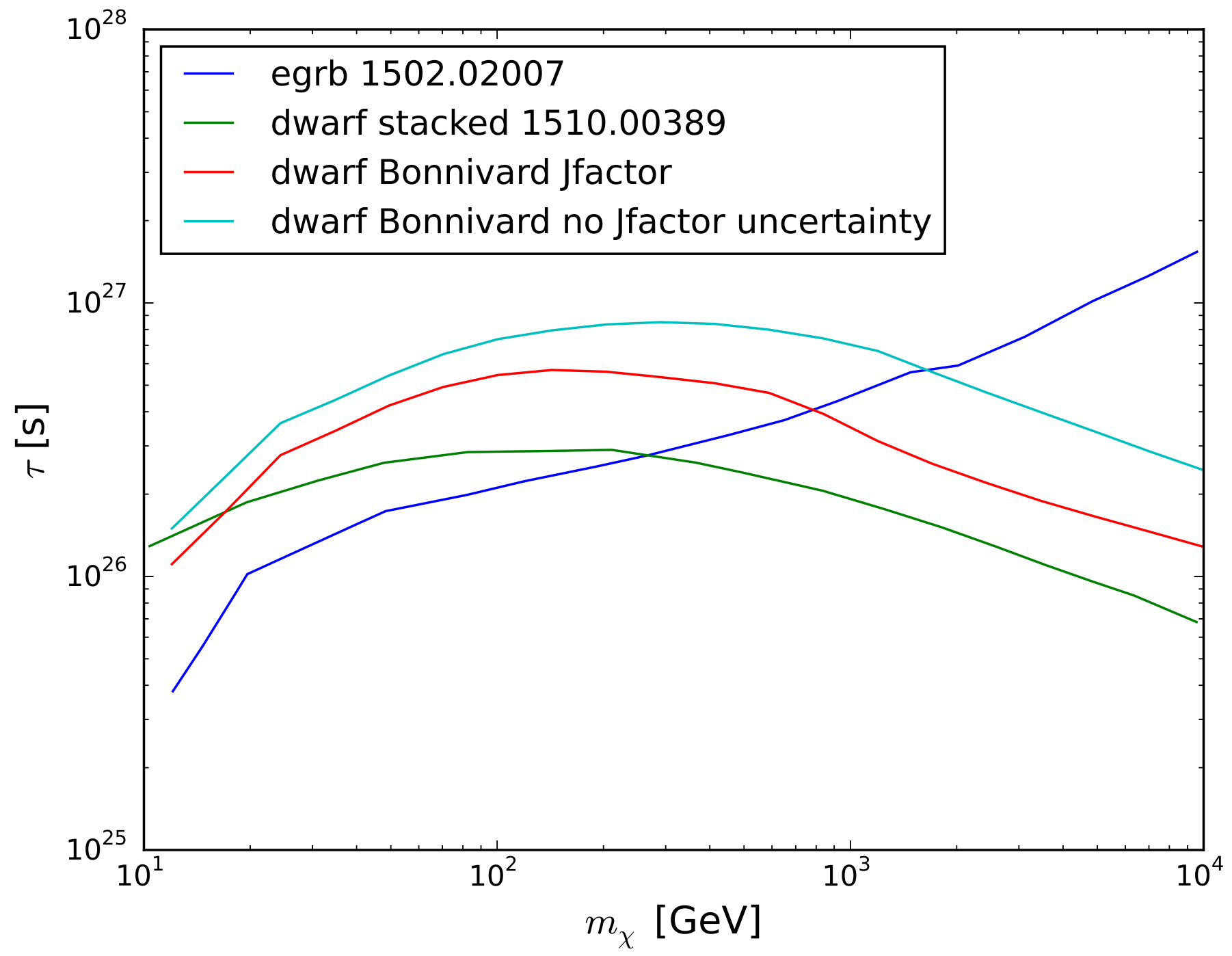

